\title{
Statistics of vacuum breakdown in the high-gradient and low-rate regime
}

\author{
Walter Wuensch, Alberto Degiovanni, and Sergio Calatroni \\ CERN, European Organization for Nuclear Research, 1211 Geneva, Switzerland \\ Anders Korsbäck* and Flyura Djurabekova \\ Helsinki Institute of Physics, University of Helsinki, P.O. Box 43, FI-00014 Helsinki, Finland \\ Robin Rajamäki \\ Aalto University, School of Electrical Engineering, P.O. Box 13000, FI-00076 AALTO, Espoo, Finland \\ Jorge Giner-Navarro \\ Instituto de Física Corpuscular (CSIC-UV), E-46071 Valencia, Spain
}

(Received 24 September 2016; published 25 January 2017)

\begin{abstract}
In an increasing number of high-gradient linear accelerator applications, accelerating structures must operate with both high surface electric fields and low breakdown rates. Understanding the statistical properties of breakdown occurrence in such a regime is of practical importance for optimizing accelerator conditioning and operation algorithms, as well as of interest for efforts to understand the physical processes which underlie the breakdown phenomenon. Experimental data of breakdown has been collected in two distinct high-gradient experimental set-ups: A prototype linear accelerating structure operated in the Compact Linear Collider Xbox $12 \mathrm{GHz}$ test stands, and a parallel plate electrode system operated with pulsed $\mathrm{DC}$ in the $\mathrm{kV}$ range. Collected data is presented, analyzed and compared. The two systems show similar, distinctive, two-part distributions of number of pulses between breakdowns, with each part corresponding to a specific, constant event rate. The correlation between distance and number of pulses between breakdown indicates that the two parts of the distribution, and their corresponding event rates, represent independent primary and induced follow-up breakdowns. The similarity of results from pulsed DC to $12 \mathrm{GHz}$ rf indicates a similar vacuum arc triggering mechanism over the range of conditions covered by the experiments.
\end{abstract}

DOI: 10.1103/PhysRevAccelBeams.20.011007

\section{INTRODUCTION}

The target accelerating gradient for the $3 \mathrm{TeV}$ center of mass energy version of Compact Linear Collider (CLIC) is $100 \mathrm{MV} / \mathrm{m}$ [1], which results in surface electric fields of around $250 \mathrm{MV} / \mathrm{m}$ in the accelerating structure [2]. The high gradient serves to limit facility size and reduce cost. In addition to operating at high gradient, the accelerating structures must also operate very stably in order to limit luminosity loss due to perturbation of the beam. Vacuum arcing in the structures, also referred to as breakdown, imparts random transverse kicks to the beam $[3,4]$. Hence, the tendency of normal conducting radio frequency (rf) structures to experience breakdown at high fields poses the main limitation on practically usable accelerating gradient. In the case of CLIC, stability requirements have resulted in the specification of a maximum BDR (breakdown rate) of $3 \times 10^{-7}$ breakdowns per pulse of $\mathrm{rf}$ power and per meter of accelerating structure [1]. This

\footnotetext{
*anders.korsback@helsinki.fi
}

Published by the American Physical Society under the terms of the Creative Commons Attribution 3.0 License. Further distribution of this work must maintain attribution to the author(s) and the published article's title, journal citation, and DOI. specification limits overall luminosity loss due to breakdown to 1 percent in the $3 \mathrm{TeV}$ machine. Other linac applications, such as XFELs and medical accelerators, require comparable combinations of high surface fields and low breakdown rate following similar rationales [5].

Within the accelerator community, numerous studies of rf breakdown have been carried out over the last two decades. Breakdown rate was identified and introduced as an explicit performance target by the NLC/JLC linear collider studies [6,7]. Studies have been made of how breakdown rate depends on accelerating gradient and pulse length [7-12], structure geometry [9,11], and structure material [8]. The phenomenon of conditioning, i.e. the gradual improvement of structure performance over operating time, has been studied (referred to as "processing" in older papers) $[6,7,12,13]$.

Beyond the accelerator community, vacuum breakdown has been studied on a more fundamental level mainly for its relevance for the design of vacuum insulation and vacuum circuit breakers. Experiments have mainly been conducted by applying either voltage pulses or DC voltage levels over pairs of parallel plate electrodes. Threshold voltage of breakdown has been the main quantity of interest, and it has been found to follow a Weibull distribution $[14,15]$. Studies 
have been done of how the threshold voltage depends on vacuum gap distance [14,16,17], electrode area [14,18,19], external circuit characteristics [17,20,21], surface coating [17], and the presence of embedded microparticles in the cathode [22].

The objective of the analysis presented in this paper is to look more closely at the statistical properties of the occurrence of breakdown, of how breakdowns occur in relation to each other in both time and space. This was initially motivated by observation in the experimental facilities at CERN that breakdown rate is not constant in time, but rather that there are more active and quiescent periods. This can be seen in Fig. 1, which shows the accumulated number of breakdowns as a function of the number of rf pulses in a TD26CC accelerating structure [2]. The slope of the curve is the momentary breakdown rate and it can be seen to vary and form steplike features. There is even an indication of self-similarity on different time scales as can be seen from the insets.

The analysis presented in this paper is novel in that it goes beyond describing breakdown occurrence by using a single variable of breakdown rate, as has been the practice in the rf accelerator field. It also extends the same analysis to breakdown between parallel plates across vacuum insulation gaps, providing an alternative perspective to the focus on threshold breakdown voltage that has been the norm for that field.

However, there are a number of results from previous work that should be mentioned for their relevance to this paper. Adolphsen [7] reported that rf breakdowns in accelerating structures tend to arrive in clusters of a disproportionate number occurring within a small time. Shioiri et al. [23] measured breakdown voltage between parallel plate electrodes, and found similar clustering. Djogo et al. [24] measured breakdown voltage between parallel plate electrodes. When they applied pulsed voltage, they obtained breakdown voltages in two distinct parts of the voltage range, each individually following a Weibull distribution. When they instead applied a steadily rising DC

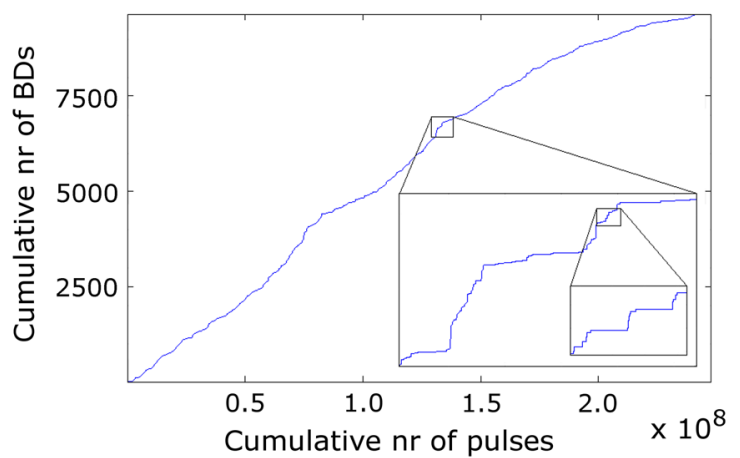

FIG. 1. Operation history of the TD26CC, cumulative number of breakdowns as a function of cumulative number of $\mathrm{rf}$ power pulses. Insets of zoom-ins show how the curve is self-similar over different time scales. level, they obtained only one Weibull distribution consistently with most other studies. Nefyodtsev et al. [25] studied pre-breakdown field emission of electrons from the cathode surface. They observed and mapped emission current densities and localized breakdowns. They found that emission sites are often unstable and vanish after a certain exposure to applied voltage. They also found that about half of all breakdowns create new emission sites close to the breakdown site, and that such newly created sites require slightly lower voltages than other sites to undergo breakdown.

There are important practical implications to the statistical properties of breakdown. For example, if structures require a temporary decrease in power to recover from a breakdown event, this will require dedicated hardware and associated linac energy maintaining algorithms to be rather active. However, if at low breakdown rate, structures can run uninterrupted following a breakdown, this would greatly simplify operation of an accelerator. Another important question is if the maximum performance state of a structure can be determined without irreversibly damaging the structure. High-gradient tests tend to be carried out by progressively increasing input power and thus accelerating gradient, but when doing so, it is difficult to determine whether maximum performance has been achieved without exceeding a possible critical value.

The statistics of breakdown are also important for understanding the underlying mechanisms of breakdown and conditioning. For example, to what extent do features created by breakdown represent sites where further breakdowns are likely to occur or do breakdowns primarily occur on "fresh" sites? Does this balance change when operating conditions, gradient and pulse length, change? Does the type of site, previous or fresh, change as conditioning progresses? The type of analysis made in this paper may also be useful for benchmarking and providing empirical validation of theoretical work on breakdown [10,26]. Theoretical understanding of breakdown remains incomplete at this time, particularly with regards to the microstructural mechanisms of the field-induced ejection of matter from the structure surface that initiates the evolution of breakdown plasma, and with regards to how the mechanisms are affected by structure material conditions. Ultimately, advances in the understanding of the materials science of breakdown could enable the development of new accelerating structures or materials that push the envelope and enable higher accelerating gradients than has previously been feasible.

This study represents an initial effort to address these and similar questions by analyzing high-gradient data and developing appropriate methods for statistical data analysis. The data has been collected by two experimental systems at CERN. The first is the CLIC prototype $\mathrm{rf}$ accelerating structure TD26CC [2], which was run at the testing facility Xbox-1 [27]. The second is a system we call 


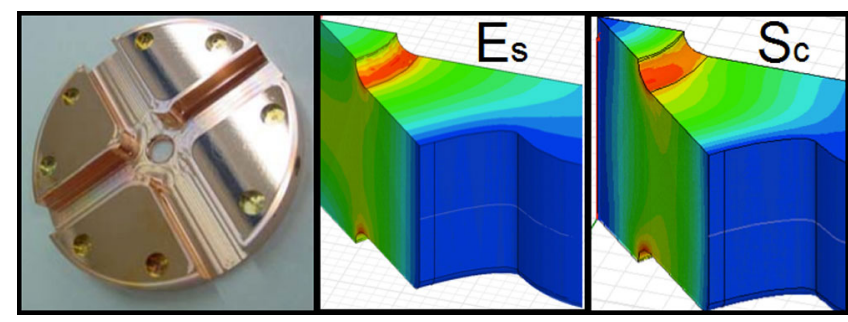

FIG. 2. Left to right: A regular TD26CC cell [2] and simulations of the electric field and modified Poynting vector inside the cell. The peak surface fields are located on the iris. Simulations courtesy of Alexej Grudiev.

the Large Electrode DC Spark System, a lower voltage system that applies pulsed DC in the $\mathrm{kV}$ range over parallel plate electrodes, providing a low-cost, high-throughput complement to rf experiments.

\section{EXPERIMENTAL METHODS}

\section{A. Rf experimental setup}

The rf measurements described in this paper were conducted on a test version of the baseline CLIC accelerating structure [1], known as the TD26CC [2]. It was tested at the klystron-based test stand Xbox-1 [27] at CERN. All measurements were done without the presence of a particle beam. The TD26CC is a constant gradient, traveling-wave structure with 26 accelerating cells, two compact coupling cells and higher-order-mode damping waveguides. The peak surface electrical field and modified Poynting vector [9] are concentrated on the cell irises, as can be seen from Fig. 2. Relevant parameters of the TD26CC are summarized in Table I.

The diagnostic tools used during rf tests in Xbox-1 are described in [28]. The main diagnostic signals are the phase and amplitude of the rf power incident on, transmitted through, and reflected back from the structure (Fig. 3). These signals are digitized using a log-detector (12 bits, $250 \mathrm{Msps}$ ) and IQ-demodulator (8 bits, $1 \mathrm{Gsps}$ ), which are connected to directional couplers before and after the structure. The occurrence of a breakdown causes a rapid increase in reflected power at the expense of transmitted power, up to full reflection of all incident rf power when the plasma arc is fully developed. The time delay between the rising edge of incident power and that of reflected power (or, alternatively, falling edge of transmitted power) is roughly proportional to distance traveled by the rf pulse

TABLE I. TD26CC structure parameters [2].

\begin{tabular}{lc}
\hline No. of cells (regular, total) & 26,28 \\
Structure length (regular, total) [mm] & 217,233 \\
Attenuation [dB] & 3.88 \\
Filling time [ns] & 67 \\
rf phase advance per cell [rad] & $2 \pi / 3$ \\
\hline \hline
\end{tabular}

from the start of the structure to the location where the breakdown occurred. Thus, this time delay can be used as a 1D position coordinate and a rough estimate of breakdown position in the structure (Fig. 3), localizing the breakdown with a resolution of approximately one or two structure cells. A detailed description of the techniques used for breakdown detection and localization can be found in [28]. For this work, our main interest in breakdown localization is to investigate spatial correlation between subsequent breakdowns.

\section{B. Pulsed DC experimental setup}

The pulsed DC experiments were conducted using an experimental setup that we have named the Large Electrode DC Spark System. It applies square voltage pulses over a
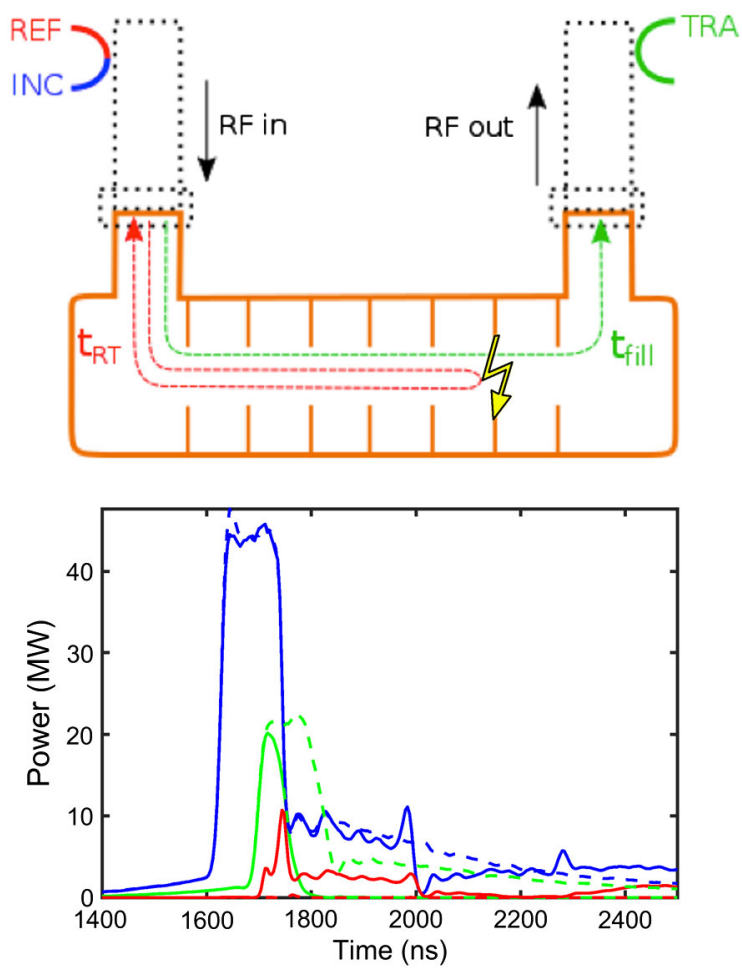

FIG. 3. Signal propagation through a traveling wave structure. Conceptual drawing of signal propagation with and without breakdown (above), example timeseries of recorded rf signals (below). In the absence of breakdown, rf power propagates through, reaching the output coupler after the filling time $\left(t_{\text {fill }}\right)$. If a breakdown happens, rf power is reflected back and reaches the input coupler after the return trip time $\left(t_{\mathrm{RT}}\right)$, roughly proportional to longitudinal breakdown position. A graph shows incident power at the input coupler (blue), transmitted power at the output coupler (green), and power reflected back to the input coupler (red). Solid lines are for a breakdown, dashed lines for a preceding nonbreakdown pulse. Breakdowns are characterized by the rise of the reflected power and the simultaneous premature fall of transmitted power. Higher-order reflections between the vacuum arc and the rf network cause oscillations in the tail ends of the signals. 
vacuum gap between two identical, interchangeable disc electrodes (diameter $62 \mathrm{~mm}$ ) facing each other, their surfaces parallel. The geometry is maintained by stacking the electrodes, separated by a cylindrical ceramic spacer resting on an outer rim apart from the gap. The height of the spacer determines the gap distance. In all DC experiments presented in this paper, a gap distance of $60 \mu \mathrm{m}$ was used. All components are precision-manufactured to micrometre tolerance.

DC voltage pulses are applied over the electrodes by using a high repetition rate experiment control system. A high-voltage DC power supply constantly charges a $200 \mathrm{~m}$ long coaxial cable acting as a pulse forming line (PFL). A DC voltage pulse is passed on from the PFL to the electrodes by opening and closing a fast, high-voltage solid state switch manufactured by Behlke. The system is capable of operating at a repetition rate of up to 1000 pulses per second, subject to power dissipation constraints. An inductive current transformer is used to measure the current going to the electrodes, and the exceeding of a threshold value used to determine the occurrence of a breakdown in the interelectrode gap. When a breakdown occurs, the PFL is quickly drained by the current across the gap, and the breakdown extinguished upon the depletion of charge stored in the PFL. The parallel-plate electrode geometry has a significant gap capacitance which is charged by the voltage pulse. An electrode and example time series of gap voltage and current are shown in Fig. 4. A bleed resistor in parallel with the electrodes is used to dissipate the charge stored in the gap capacitance between one pulse and the next. The choice of bleed resistance affects system operation. A low bleed resistance dissipates stored charge quickly, resulting in a quick voltage drop after the switch has opened, and a roughly square voltage pulse. However, a low bleed resistance increases the power dissipation in the switch, limiting repetition rate. Hence, there is a tradeoff between repetition rate and pulse shape integrity. As this study is not primarily concerned with pulse length effects, we prioritized repetition rate and accepted a pulse shape with a slow roll-off. The choice was made partly due to the secondary significance of pulse length effects for this study, and partly due to observed low prevalence of breakdown far out in the roll-off. Detailed descriptions of the system and related issues can be found in $[29,30]$.

\section{System operation and data collection}

Four data sets collected by the experimental setups are presented, referred to by the letters A through D.

Data set A is the data from which Fig. 1 was created. It was collected by operating the TD26CC structure in Xbox-1 in conditioning mode. The process of producing the structure is found in [31]. In brief, structure cells (Fig. 2) are annealed, stacked and hydrogen bonded, fusing the cells together and softening the copper in the process.
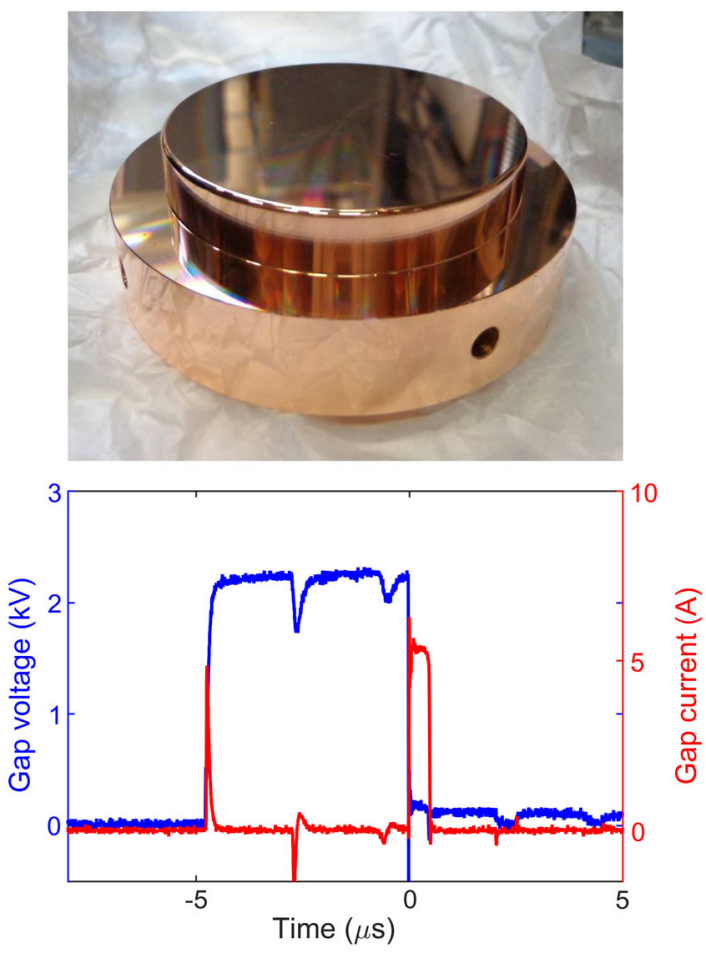

FIG. 4. One of a pair of electrodes used for pulsed DC experiments (above), and example time series of gap voltage and current when a breakdown occurs (below). Vacuum gap is formed between the top surface of the electrode, and the same surface on an identical top electrode facing it symmetrically. The outer rim of the electrode supports the ceramic spacer that holds the electrodes apart and determines the vacuum gap distance. Electrode edges are gently rounded (rounding radius $\gg$ gap distance) to prevent breakdown through the ceramic, and to prevent field enhancement from sharp edges. When the switch is closed, gap voltage (blue) rises to its top value, and gap current (red) briefly spikes as the gap capacitance is charged. During the pulse, gap voltage ripples due to reflections in the PFL. When a breakdown happens $(t=0)$, gap voltage rapidly drops as the breakdown plasma short-circuits the gap, while gap current rises to a value determined by the circuit resistance in series with the gap. Upon depletion of charge in the PFL, gap current drops and the plasma burns out.

Conditioning is the development of resistance to breakdown over accumulated total exposure to voltage pulses, and can be described either in terms of a decrease in breakdown rate for a given input power and pulse length, or an increase in the input power and/or pulse length that produce a given breakdown rate. It is mainly the latter description which is of interest for us. A description of the conditioning algorithm is found in [27]. In summary, the structure was continuously pulsed at a repetition rate of $50 \mathrm{~Hz}$, with input power regulated according to breakdown behavior. After each breakdown, pulsing was stopped to allow system recovery. Pulsing was resumed by ramping up input power step-wise with a proportional feedback loop asymptotically toward a set value. The set value itself was 
increased whenever the system had a certain number (typically 15000 to 18000) consecutive pulses without breakdown since last breakdown or increase of power, and decreased whenever a breakdown occurred within a certain number (typically 4500 to 6000) of pulses since last breakdown. Control algorithm parameters were manually adjusted by the system operator. Pulse length was 50 ns at the start of the run, and was increased at operator discretion in $50 \mathrm{~ns}$ increments up to a final value of $250 \mathrm{~ns}$.

The breakdown localization capability of Xbox-1 was used to obtain a position coordinate for each breakdown along the longitudinal axis of the structure.

The same TD26CC structure was previously high-power tested in Xbox-1 in 2013. Afterwards, the structure was exposed to air for approximately six weeks and rebaked in $650{ }^{\circ} \mathrm{C}$ in order to remove excess hydrogen before retesting. The results of the 2013 run are presented in [32].

Data sets B, C and D were collected by the Large Electrode DC Spark System, in that order. They were all collected using the same pair of copper electrodes. These electrodes had not gone through the material treatment that the TD26CC structure used for data set A had, and were thus of harder copper. Each data set was collected by using a chosen, fixed voltage pulse length and amplitude. Sequences of voltage pulses were sent until a breakdown occurred, at which point the pulsing was temporarily stopped and the number of pulses in the sequence was recorded, thus providing statistics on overall breakdown rate and on the distribution of number of pulses between breakdowns. To mimic the operation of Xbox-1 in its collection of data set A, each sequence was started by ramping up voltage asymptotically toward the set value so that $90 \%$ of the final voltage would be reached after about 700 pulses, and consequently $99 \%$ of it after about 1400 pulses. The ramping was carried out by making use of the charging of the capacitance of the PFL.

The input parameters used in the measurement runs are listed in Table II.

\section{RESULTS}

\section{A. Distribution of number of pulses between breakdowns}

For each data set, a numerical probability density function (PDF) of number of pulses between one

TABLE II. Input parameters data sets A to D.

\begin{tabular}{lcccc}
\hline \hline Data set & $\mathrm{A}$ & $\mathrm{B}$ & $\mathrm{C}$ & $\mathrm{D}$ \\
\hline Voltage $[\mathrm{kV}]$ & $n / a$ & 2.3 & 2.8 & 4.2 \\
Pulse length $[\mu \mathrm{s}]$ & $n / a$ & 6.0 & 3.5 & 2.0 \\
Total nr BDs & 9662 & 30108 & 1537 & 3200 \\
Total nr pulses $\left[\times 10^{6}\right]$ & 246 & 784 & 139 & 87 \\
Overall BDR $\left[\times 10^{-5}\right]$ & 3.923 & 3.840 & 1.10 & 3.690 \\
\hline \hline
\end{tabular}

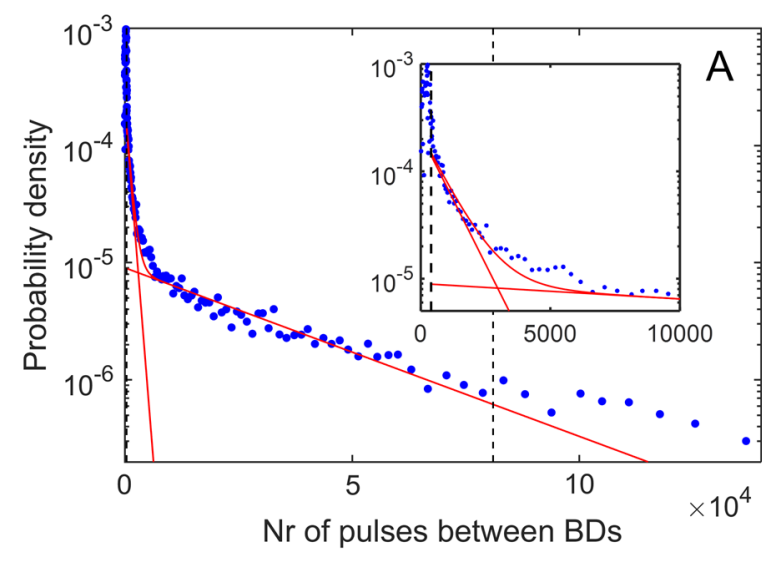

FIG. 5. Distribution of number of pulses between breakdowns and two-exponential fit, data set A. Inset shows a zoom-in of the start of the distribution.

breakdown and the next was computed. Figures 5, 6, 7, and 8 show the resulting PDFs.

Despite the differences between the measurements that produced the data sets, pulsed rf and pulsed DC, the resulting PDFs all have the same overall shape of a sum of two exponential decreases. To highlight the functional dependence, the PDFs are plotted with a linear $\mathrm{x}$-axis and a logarithmic y-axis, whereby an exponential decrease shows as a downward-sloping line, and a sum of two exponentials as two lines connected by a rounded kink. To each PDF, a sum of two exponentials $\operatorname{PDF}(n)=$ $\operatorname{Aexp}(-\alpha n)+\operatorname{Bexp}(-\beta n)$ was fit, with $n$ being the number of pulses between breakdowns, and with $\alpha<\beta$ as a convention so that the first term will always correspond to the long-term part of the distribution with the gentler slope and the second term to the short-term part with the steeper slope at the start of the distribution. Each PDF is plotted together with its two-exponential fit, broken down into its two terms. Part of the data from the lower and upper ends of the distributions was excluded from the fits, the range of

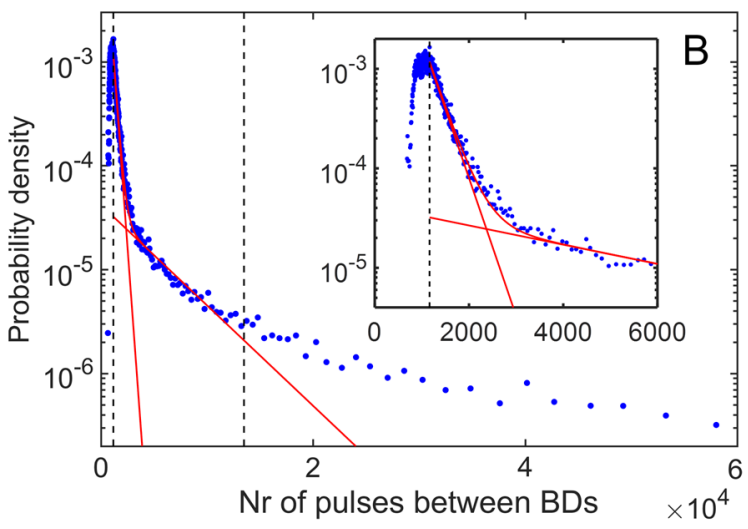

FIG. 6. Distribution of number of pulses between breakdowns and two-exponential fit, data set B. Inset shows a zoom-in of the start of the distribution. 


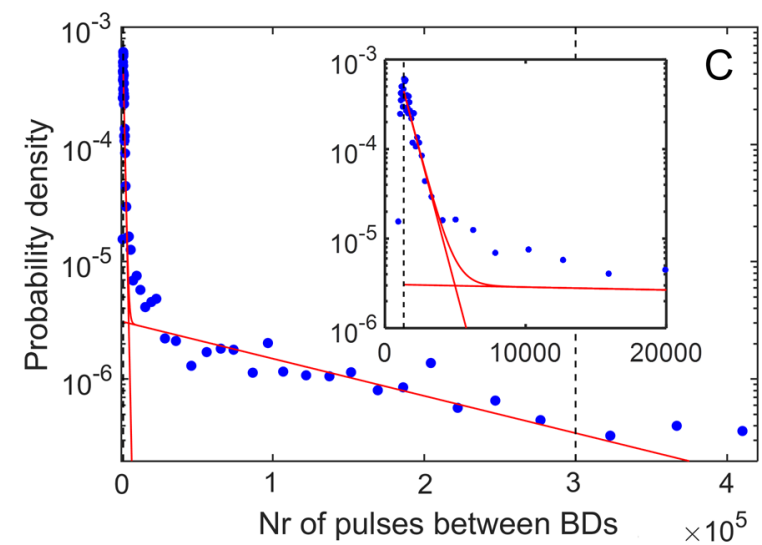

FIG. 7. Distribution of number of pulses between breakdowns and two-exponential fit, data set $\mathrm{C}$. Inset shows a zoom-in of the start of the distribution.

each fit is delineated by the dashed vertical lines. The exclusion from the low end was made to account for the fact that after each breakdown, voltage was ramped up from zero asymptotically toward its set value, causing the first pulses to be of lower amplitude than the subsequent and less likely to cause breakdown. For data sets B, C, and $\mathrm{D}$, the lower bound was set at the peak of the PDF. For data set A, it was selected by hand. This was done because in the measurement that produced data set $\mathrm{A}, \mathrm{rf}$ power was increased stepwise in the ramp-up, introducing a statistical artifact as many breakdowns happened immediately after a step. The exclusion of data from the upper end was due to a failure of the fit at the upper tail end of the distribution, so rather than attempt a full explanation of the distribution, we settle for a description that works satisfactorily for the great majority of the data, around 90\% depending on the data set. The origin of extremely long intervals between breakdowns will be revisited in the future. The choice of where to place the upper bound of the fit is somewhat arbitrary, so out of consistency we set it

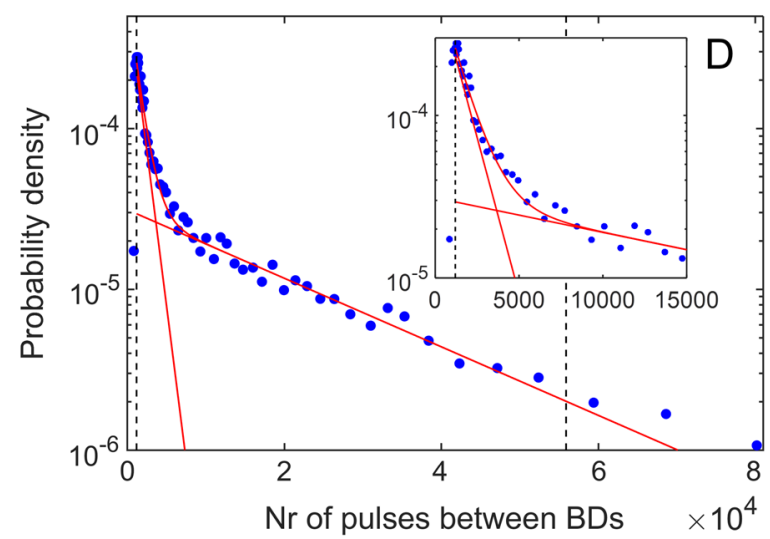

FIG. 8. Distribution of number of pulses between breakdowns and two-exponential fit, data set D. Inset shows a zoom-in of the start of the distribution. in each case so that the top 10\% of the PDF was excluded. Each plot was drawn excluding the top 5\% in order to show the increasing divergence between the fit and the PDF at the tail end but not to obscure the distribution by unnecessarily extending the $\mathrm{x}$-axis.

An exponentially decreasing PDF for times between events indicates that the events follow Poisson statistics, which describe events that occur at a constant event rate independently of each other. Thus, a PDF which is a sum of two exponentials suggests that there are, instead, two different system states, both with a specific, constant event rate.

One possible physical interpretation of the observed twopart distributions is that there are two kinds of breakdown events: Primary breakdowns which occur randomly and independently at some point on the high-field surface, and follow-up breakdowns which occur as a direct result of a previous breakdown. The mechanism of the induction of a follow-up breakdown might for example be surface modification, such as increase in roughness or the formation of high aspect ratio peaks in the vicinity of a breakdown spot. Such features would be particularly susceptible to breakdown, which would show as an enhanced momentary breakdown rate immediately after an inducing breakdown (which could either be a primary breakdown or a previous follow-up breakdown). Surface modification in the vicinity of a breakdown is a known phenomenon that has been studied through microscopy [33] and materials physics simulation [34].

We will now explore this hypothesis by examining the properties of the distributions. A number of relevant parameters are presented in Table III. Dividing the total number of breakdowns during the run by the total number of pulses during it gives the overall breakdown rate (BDR).

The fit coefficients $\alpha$ and $\beta$ are worth examining and discussing for their physical significance. For a purely Poissonian distribution consisting of a single exponential decrease, the coefficient of the exponential is the event rate. Primary breakdowns are such Poissonian events. Thus it is relevant to call $\alpha$ the "primary BDR," as it is the probability

TABLE III. Parameters of data sets A to D, their PDFs and the two-exponential-fits to them.

\begin{tabular}{lcccc}
\hline \hline Data set & $\mathrm{A}$ & $\mathrm{B}$ & $\mathrm{C}$ & $\mathrm{D}$ \\
\hline Voltage $[\mathrm{kV}]$ & $n / a$ & 2.3 & 2.8 & 4.2 \\
Pulse length $[\mu \mathrm{s}]$ & $n / a$ & 6.0 & 3.5 & 2.0 \\
Total nr BDs & 9662 & 30108 & 1537 & 3200 \\
Total nr pulses $\left[\times 10^{6}\right]$ & 246 & 784 & 139 & 87 \\
Overall BDR $\left[\times 10^{-5}\right]$ & 3.923 & 3.840 & 1.10 & 3.690 \\
Primary BDR $\alpha\left[\times 10^{-5}\right]$ & 3.307 & 22.22 & 0.7302 & 4.903 \\
Follow-up BDR $\beta\left[\times 10^{-3}\right]$ & 1.107 & 2.187 & 1.371 & 0.8851 \\
$\beta / \alpha$ & 33.47 & 14.34 & 187.8 & 18.05 \\
Nr followups / Nr primaries & 1.336 & 4.042 & 0.9382 & 0.5912 \\
Primary BD follow-up rate & 0.4031 & 0.2946 & 0.4255 & 0.2438 \\
\hline \hline
\end{tabular}


that any individual pulse will cause a primary breakdown, as well as the expectation value for the total number of primary breakdowns during a measurement run divided by the total number of pulses during the run. Consistently, we will call $\beta$ the "follow-up BDR," as it is the coefficient of the fit term corresponding to follow-up breakdowns. It is the probability that any individual pulse will cause a follow-up breakdown, if the system is in a state of enhanced breakdown susceptibility due to a recent breakdown. However, since follow-up breakdowns are not independent events but only occur in such a state of enhanced breakdown susceptibility, $\beta$ does not by itself contain any information about the expected number of follow-up breakdowns during a measurement run. To deduce that, one would need information about the statistics of breakdown clustering, i.e., the distribution of number of followup breakdowns after a primary breakdown. Clustering statistics is a subject that will be revisited in the future.

$\alpha$ and overall BDR tend to be in the same order of magnitude. In the cases of data sets B and D, $\alpha$ is larger than overall BDR. This is not inconsistent since $\alpha$ is a fit parameter while overall BDR was obtained by counting breakdowns and pulses, whereby the breakdowns on the tail end of the PDF excluded from the fit contribute disproportionately to the total number of pulses. We note that the ratio between the two breakdown rates, $\beta / \alpha$, varies by about an order of magnitude among the data sets.

We can, with reasonable accuracy, identify breakdowns as either primary or follow-up by using the intersect between the two terms of the fit as a divider, classifying all breakdowns happening before this threshold number of pulses as follow-ups and all happening after it as primaries. By doing so, we can obtain a value for the ratio between the total number of breakdowns of both kinds, referred to as "Nr followups / Nr primaries" in Table III. We can also obtain a value for the probability that a primary breakdown will be followed by at least one follow-up breakdown, we refer to this quantity as "Primary BD follow-up rate." One would expect these two quantities to be positively correlated and we note that they generally are, but not universally so. Data set B has by far the largest ratio "Nr followups / Nr primaries" but the second-lowest "Primary BD follow-up rate." This is counterintuitive but not inconsistent. A primary breakdown can be followed by a variable number of follow-ups, either through the primary inducing multiple follow-ups to begin with, or by the follow-ups inducing further follow-ups in a cascadelike manner. In the case of data set B, relatively few primaries induced follow-ups, but those that did were often followed by long sequences of follow-ups.

A relevant question is whether the distribution is stable over time. Data set B offers the possibility to investigate distribution stability, as it is large enough to be divided into subsets and was collected at constant voltage and pulse length (unlike data set A, where rf power was

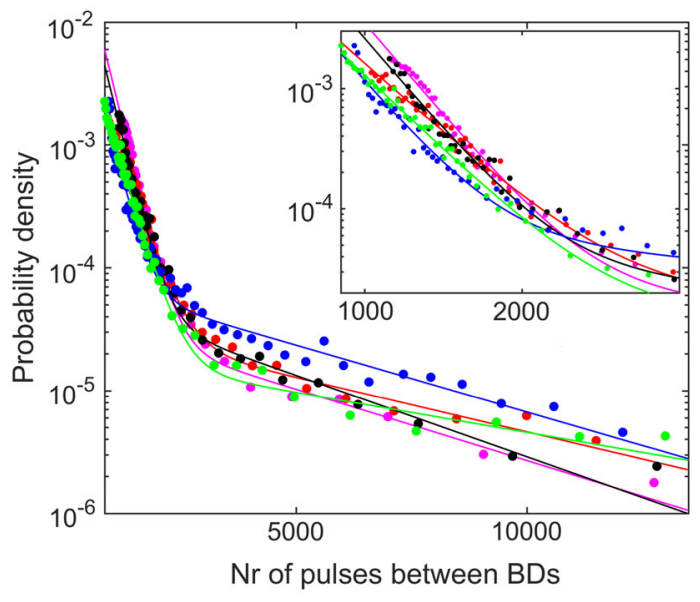

FIG. 9. Distribution of number of pulses between breakdowns for five subsets (in order: pink, red, black, blue, green) of data set $\mathrm{B}$, and a two-exponential fit for each. Inset shows a zoom-in of the start of the distribution.

feedback-controlled according to breakdown behavior). Data set B was divided into five sequential subsets, all containing 6021 or 6022 breakdowns. A PDF of number of pulses to breakdown was computed for each subset. A twoexponential fit made to each subset, using the same fit $\mathrm{X}$ axis upper bound as for the entire set. The PDFs and their fits are shown in Fig. 9. The parameters of the fits are listed in Table IV.

We see that all subset PDFs show the same twoexponential shape as the PDF of the entire set, though the slopes of both parts of each fit and the overall breakdown rates vary between subsets. All parameters seem to fluctuate rather than follow a long-term trend.

That the overall shape of the distribution is unchanged when dividing the data into subsets supports our interpretation of the data. The fluctuation of $\alpha$ and $\beta$ could be due to physical alteration of the electrodes occurring over the long term as pulses are applied, or due to fluctuations in external experimental conditions that we have not identified. The former explanation is likely. It is consistent with the experience of rf breakdown behavior, which as mentioned earlier is that structures go through more active and quiescent periods. Figure 1 shows a staircase-like pattern on the two zoom levels. The steps on the innermost zoom level would thus be caused by primary breakdowns followed by clusters of follow-up breakdowns, while the

TABLE IV. Overall, primary $(\alpha)$ and follow-up $(\beta)$ breakdown rate of data set B and its subsets (Fig. 9).

\begin{tabular}{lcccccc}
\hline \hline Data set & $\mathrm{B}$ & $\mathrm{B} 1$ & $\mathrm{~B} 2$ & $\mathrm{~B} 3$ & $\mathrm{~B} 4$ & $\mathrm{~B} 5$ \\
\hline Overall BDR $\left(\times 10^{-5}\right)$ & 3.840 & 14.30 & 5.788 & 14.49 & 1.156 & 7.977 \\
$\alpha\left(\times 10^{-5}\right)$ & 22.22 & 26.73 & 20.38 & 30.51 & 25.09 & 14.99 \\
$\beta\left(\times 10^{-3}\right)$ & 3.187 & 3.556 & 2.702 & 3.558 & 3.548 & 2.909 \\
$\beta / \alpha$ & 14.34 & 13.30 & 13.26 & 11.66 & 14.14 & 19.41 \\
\hline \hline
\end{tabular}


steps on the outer zoom level would be due to fluctuations of $\alpha$ and/or $\beta$.

Postulating that primary breakdowns are Poissonian events with an event rate that slowly fluctuates would also provide an explanation to why the two-exponential fit fails at the upper tail end of the PDF. The disproportionate occurrence of extremely long series of pulses between breakdowns could be the result of $\alpha$ fluctuating down an entire order of magnitude or more from the values we obtain from the PDFs. Such fluctuations would not be directly detected in a simple subset analysis like this, as there would be too few breakdowns in total during such a period to get proper statistics for a subset. The fact that overall breakdown rate varies greatly between subsets, and is consistently lower than $\alpha$ but shows no clear correlation to it, suggests that such quiescent periods occur within the subsets. Visual inspection of the data reinforces this impression. Deeper study of the question of quiescent periods and fluctuating event rates is also one we defer to future work.

\section{B. Spatio-temporal correlation of breakdowns}

If the hypothesis of primary and follow-up breakdowns is the correct, then one would expect follow-up breakdowns to occur at or near the location of the preceding breakdown. As Xbox-1 has the capability to localize breakdowns longitudinally as described in Sec. II A, the degree of spatial correlation between successive breakdowns in data set A was studied. Figure 10 shows the longitudinal distance between the sites of successive breakdowns, plotted against the number of pulses between them. Each point is derived from a pair of two successive breakdowns. A small vertical spread occurs when successive breakdowns tend to occur near each other, a large vertical spread occurs when they tend to occur farther apart. A tendency for vertical spread to increase as number of pulses between the breakdowns increases can be seen. To visualize the tendency, bounds are drawn containing the middle $25 \%, 50 \%$, and $75 \%$ of the data points in given ranges of $\mathrm{x}$-values. Below the scatter plot, the PDF of number of pulses to breakdown is given for data set $\mathrm{A}$, together with the two-exponential fit. This is the same plot as Fig. 5, but plotted with a logarithmic x-axis common with the scatter plot. The relative magnitudes of the two terms of the fit can be used to obtain the probability that a given breakdown is a follow-up breakdown, a probability that is a function of the number of pulses between the breakdown and the preceding one. In the PDF, the point at which the ratio of the two terms of the fit is such that the probability of a breakdown to be a follow-up is $5 \%$ has been marked. The corresponding number of pulses between breakdowns has been marked with a vertical red line in the scatter plot. We note an almost monotonic increase in vertical spread, and thus a decrease in the strength of the spatial correlation, up to this line. Soon afterward, the

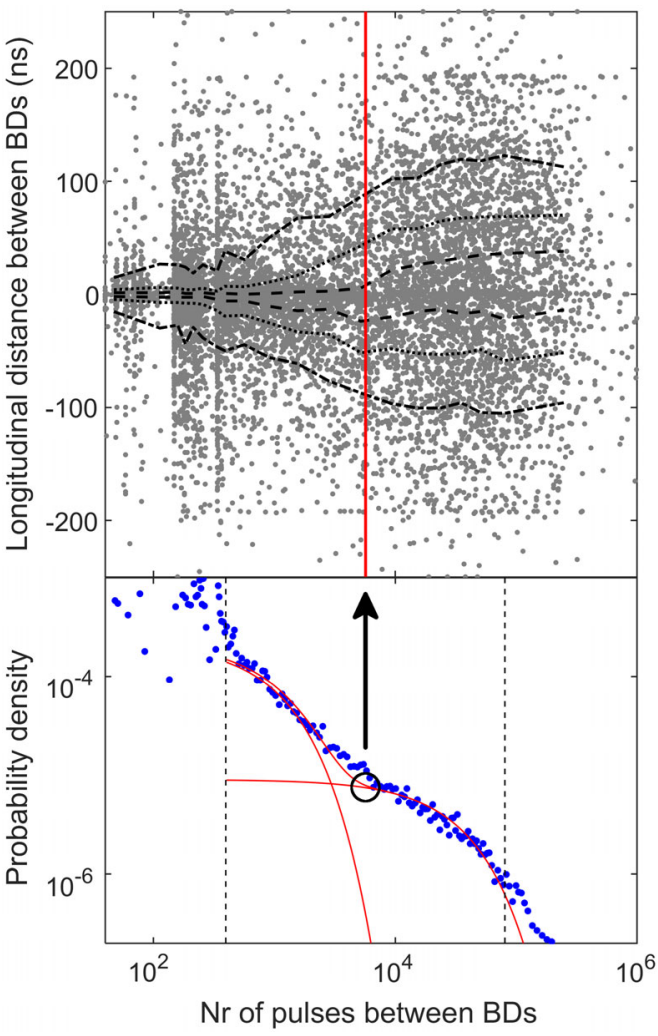

FIG. 10. Data set A, comparison of spatio-temporal correlation (above) and distribution of number of pulses between breakdowns (below), plotted on a common x-axis. Top graph shows longitudinal distance (in units of signal travel time) between each pair of successive breakdowns, as a function of number of pulses between them. Dashed, dotted, and dash-dot lines show, respectively, the intervals containing the middle $25 \%, 50 \%$, and $75 \%$ of the data. Bottom graph is the distribution of number of pulses between breakdowns, i.e., the same as Fig. 5, but drawn with a logarithmic $\mathrm{x}$-axis. The black circle marks the value of number of pulses between breakdowns for which there is, according to the two-exponential fit, a $95 \%$ probability that a breakdown is a primary breakdown rather than a follow-up of the preceding one. This value of number of pulses between breakdowns is marked as a vertical red line in the upper graph.

increase in spread levels off. This consistency between the spatial correlation and the PDF strongly supports the hypothesis that the origin of the two-exponential distribution is primary and follow-up breakdowns.

\section{Comparison with other studies}

Nefyodtsev et al. [25] found that about half of all breakdowns create new field emission sites in their vicinity, and that these sites often cause further breakdowns. This provides a mechanism for the two types of breakdowns and the spatio-temporal correlation of breakdown that we have identified. As seen from Table III, the proportion of primary breakdowns that cause follow-up breakdowns varies between $24 \%$ and $43 \%$ in our data. Nefyodtsev et al. 
found that field emission sites are often unstable. From that, it would naturally follow that some emission sites created by breakdown would collapse rather than cause a follow-up breakdown. If certain ways of applying voltage are more prone to cause such collapse, that would explain why the strength of the tendency for breakdowns to cause follow-up breakdowns varies between different measurements and sets of input parameters.

Nefyodtsev et al. also found out that the new emission sites created by a breakdown are more susceptible to breakdown than the site that caused the original breakdown. This showed in their experiments as a lower threshold voltage of breakdown, and in our experiments as values of $\beta$ that are larger than $\alpha$ by an order of magnitude or more.

The comparison of our results with those of Nefyodtsev et al. offer another interpretation of the results reported by Djogo et al. [24]. They obtained values of breakdown voltage clustered in two regions of the voltage range when they applied pulsed voltage over a vacuum gap. They attributed this result to two different mechanisms being at work, one on the cathode and another on the anode. We suggest that, instead, the breakdowns occurring at the higher voltage were primary breakdowns and those at the lower voltage were follow-up breakdowns. When Djogo et al. performed the measurement with a steadily rising DC voltage level rather than by pulsing, they only obtained one cluster of breakdown voltage values rather than two. This could be explained by postulating that the rising DC voltage level effectively inhibited follow-up breakdown by collapsing the new emission sites created by breakdowns.

\section{CONCLUSIONS}

We provide a simple, quantitative description of the statistics of the occurrence of breakdown in the highgradient, low breakdown rate regime. The number of pulses between subsequent breakdowns follows a distribution with a long- and a short-term part, each following approximately an exponential decrease. This indicates the existence of two system states, each with a specific, constant event rate.

Through an analysis of the positions at which breakdowns occur in a structure, it has been shown that breakdowns that happen close together in time tend to also happen close together in space. This has enabled identification of two kinds of breakdown events corresponding to the aforementioned system states. The long-term part of the distribution has been identified to correspond to what we call primary breakdowns, independent Poissonian events occurring at the underlying breakdown rate of a surface subjected to high electric field. The short-term part of the distribution has been identified to correspond to what we call follow-up breakdowns, events caused by a previous breakdown.

These observations and generalizations appear in two rather different experimental setups-a $12 \mathrm{GHz}$ rf accelerating structure and a pulsed DC parallel electrode system. In the latter case, the distinct two-part shape of the distribution further held true over three different combinations of input voltage and pulse length, despite significant variation in the parameters of the resulting distributions and the relative number of breakdowns in the long- and shortterm parts of them. Hence, the analysis indicates that the underlying trigger mechanism which drives the probability of breakdown in the low-rate regime is similar over a wide range of conditions and parameters.

This is a significant result for fundamental studies of the mechanisms of breakdown. For the specific field of breakdown studies in the context of accelerator technology development, it validates the use of pulsed DC systems as a low-cost, high-throughput complement to rf experiments on accelerating structures.

Our results are consistent and mutually complementary with cited previous work by other groups. Our results provide new interpretations of results reported by others. In combination, our results and those cited provide an initial indication that the induction of follow-up breakdown can be prevented or mitigated by suitable operational algorithms.

These observations and analysis tools will now be used to extend the analysis of for example breakdown rate dependence as a function of gradient, pulse length, and conditioning state. They will also be used to develop improved conditioning and operational algorithms.

\section{ACKNOWLEDGMENTS}

The authors wish to extend their sincere thanks to past and present CERN colleagues in the structure fabrication teams, Xbox teams, and the DC breakdown lab. This work was made possible by the use of high-quality structures, persistent long-term development of the experimental setups, and well-controlled long-term running of experiments.

[1] M. Aicheler, P. Burrows, M. Draper, T. Garvey, P. Lebrun, K. Peach, N. Phinney, H. Schmickler, D. Schulte, N. Toge et al., CERN Technical Report No. CERN-2012-007, 2012.

[2] A. Grudiev and W. Wuensch, Design of the CLIC main linac accelerating structure for CLIC conceptual design report, in Proceedings of the 25th International Linear Accelerator Conference, LINAC-2010, Tsukuba, Japan (KEK, Tsukuba, Japan, 2010), p. 211.

[3] A. Palaia, Ph.D. thesis, Uppsala University, 2013.

[4] V. A. Dolgashev and T. Raubenheimer, Simulation of RF Breakdown Effects on NLC Beam, in Proceedings of LINAC2004, Lübeck, Germany (DESY, Hamburg, Germany, 2004), p. 395.

[5] S. Verdú Andrés, Ph.D. thesis, University of Valencia, 2012. 
[6] C. Adolphsen, W. Baumgartner, K. Jobe, F. Le Pimpec, R. Loewen, D. McCormick, M. Ross, T. Smith, J. Wang, and T. Higo, Processing Studies of X-band Accelerator Structures at the NLCTA, in Proceedings of the 19th Particle Accelerator Conference, Chicago, IL, 2001 (IEEE, Piscataway, NJ, 2001), p. 478.

[7] C. Adolphsen, SLAC Technical Report No. SLAC-PUB9906, 2003.

[8] S. Dobert, C. Adolphsen, G. Bowden, D. Burke, J. Chan, V. Dolgashev, J. Frisch, K. Jobe, R. Jones, J. Lewandowski et al., High gradient performance of NLC/GLC X-band accelerating structures, in Proceedings of the 21st Particle Accelerator Conference, Knoxville, TN, 2005 (IEEE, Piscataway, NJ, 2005), p. 372.

[9] A. Grudiev, S. Calatroni, and W. Wuensch, New local field quantity describing the high gradient limit of accelerating structures, Phys. Rev. ST Accel. Beams 12, 102001 (2009).

[10] K. Nordlund and F. Djurabekova, Defect model for the dependence of breakdown rate on external electric fields, Phys. Rev. ST Accel. Beams 15, 071002 (2012).

[11] V. Dolgashev, Study of basic breakdown phenomena in high gradient vacuum structures, FR105, in Proceedings of the 25th International Linear Accelerator Conference, LINAC-2010, Tsukuba, Japan (KEK, Tsukuba, Japan, 2010).

[12] T. Higo, T. Abe, Y. Arakida, S. Matsumoto, T. Shidara, T. Takatomi, M. Yamanaka, A. Grudiev, G. Riddone, and W. Wuensch, Comparison of High-Gradient Performance in Varying Cavity Geometries, in Proceedings of the 4th International Particle Accelerator Conference, IPAC2013, Shanghai, China, 2013 (JACoW, Shanghai, China, 2013).

[13] A. Degiovanni, W. Wuensch, and J. Giner Navarro, Comparison of the conditioning of high gradient accelerating structures, Phys. Rev. Accel. Beams 19, 032001 (2016).

[14] H. Toya, N. Ueno, T. Okada, and Y. Murai, Statistical property of breakdown between metal electrodes in vacuum, IEEE Transactions on Power Apparatus and Systems PAS-100, 1932 (1981).

[15] H. Toya, T. Hayashi, Y. Shinozaki, and Y. Murai, Statistical property of breakdown between electrode and shield in high-voltage vacuum interrupter, IEEE Trans. Plasma Sci. 13, 311 (1985).

[16] Y. Murai, H. Toya, and T. Nitta, Statistical property of the breakdown of vacuum circuit breakers and its influence on the surge generation in capacitive and reactive current interruption, IEEE Transactions on Power Apparatus and Systems PAS-98, 232 (1979).

[17] K. Watanabe, M. Mizuno, Y. Ohara, M. Tanaka, K. Kobayashi, E. Takahashi, and T. Uede, dc voltage holding experiments of vacuum gap for high-energy ion sources, J. Appl. Phys. 72, 3949 (1992).

[18] H. Toya, T. Hayashi, M. Yorita, and Y. Murai, Numerical modeling to evaluate the withstand voltage of a HV vacuum interrupter, IEEE Trans. Electr. Insul. 24, 21 (1989).

[19] Y. Zhang, Z. Liu, S. Cheng, L. Yang, Y. Geng, and J. Wang, Influence of contact contour on breakdown behavior in vacuum under uniform field, IEEE Trans. Dielectr. Electr. Insul. 16, 1717 (2009).
[20] B. Mazurek and J. Cross, An energy explanation of the area effect in electrical breakdown in vacuum, IEEE Trans. Electr. Insul. 22, 341 (1987).

[21] J. Cross and B. Mazurek, Effect of area on surface flashover voltage in vacuum, IEEE Trans. Electr. Insul. 23, 43 (1988).

[22] W. F. Rieder, M. Schussek, W. Glatzle, and E. Kny, The influence of composition and $\mathrm{Cr}$ particle size of $\mathrm{Cu} / \mathrm{Cr}$ contacts on chopping current, contact resistance, and breakdown voltage in vacuum interrupters, IEEE Trans. Components, Hybrids, Manuf. Technol. 12, 273 (1989).

[23] T. Shioiri, T. Kamikawaji, K. Yokokura, E. Kaneko, I. Ohshima, and S. Yanabu, Dielectric breakdown probabilities for uniform field gap in vacuum, in Discharges and Electrical Insulation in Vacuum, 2000. Proceedings. ISDEIV. XIXth International Symposium on (IEEE, New York, 2000), Vol. 1, p. 17.

[24] G. Djogo and P. Osmokrovic, Statistical properties of electrical breakdown in vacuum, IEEE Trans. Electr. Insul. 24, 949 (1989).

[25] E. Nefyodtsev, S. Onischenko, D. Proskurovsky, and A. Batrakov, Observation of pre-breakdown emission sites and breakdowns of vacuum gaps under short-pulsed testing, IEEE Trans. Dielectr. Electr. Insul. 18, 929 (2011).

[26] A. S. Pohjonen, F. Djurabekova, A. Kuronen, S. P. Fitzgerald, and K. Nordlund, Analytical model of dislocation nucleation on a near-surface void under tensile surface stress, Philos. Mag. 92, 3994 (2012).

[27] N. Catalan-Lasheras, A. Degiovanni, S. Dobert, W. Farabolini, J. Kovermann, G. McMonagle, S. Rey, I. Syratchev, L. Timeo, W. Wuensch et al., Experience Operating an X-band High-Power Test Stand at CERN, in Proceedings of IPAC2014, Dresden, Germany (JACoW, Geneva, Switzerland, 2014), p. 2288.

[28] A. Degiovanni, S. Dobert, W. Farabolini, I. Syratchev, W. Wuensch, J. Giner-Navarro, J. Tagg, and B. Woolley, Diagnostics and analysis techniques for high power $\mathrm{X}$-band accelerating structures, in Proceedings of LINAC2014, Geneva, Switzerland (JACoW, Geneva, Switzerland, 2014), p. 490.

[29] N. C. Shipman, Ph.D. thesis, The University of Manchester, Manchester, UK, 2015.

[30] R. Soares, W. Wuensch, J. Kovermann, S. Calatroni, and M. Barnes, A $12 \mathrm{KV}, 1 \mathrm{KHZ}$, pulse generator for breakdown studies of samples for CLIC RF accelerating structures, in Proceedings of IPAC2012, New Orleans, 2012 (IEEE, Piscataway, 2012), THPPC061.

[31] J. W. Wang, J. Lewandowski, J. Van Pelt, C. Yoneda, G. Riddone, D. Gudkov, T. Higo, T. Takatomi et al., Fabrication technologies of the high gradient accelerator structures at $100 \mathrm{MV} / \mathrm{m}$ range, in Proceedings of the International Particle Accelerator Conference, Kyoto, Japan (ICR, Kyoto, 2010), THPEA064.

[32] A. Degiovanni, S. Dobert, W. Farabolini, A. Grudiev, J. Kovermann, E. Montesinos, G. Riddone, I. Syratchev, R. Wegner, W. Wuensch et al., High-Gradient Test Results From a CLIC Prototype Accelerating Structure: TD26CC, in Proceedings of IPAC2014, Dresden, Germany (JACoW, Geneva, Switzerland, 2014) p. 2285. 
[33] H. Timko, F. Djurabekova, K. Nordlund, L. Costelle, K. Matyash, R. Schneider, A. Toerklep, G. Arnau-Izquierdo, A. Descoeudres, S. Calatroni et al., Mechanism of surface modification in the plasma-surface interaction in electrical arcs, Phys. Rev. B 81, 184109 (2010).
[34] F. Djurabekova, J. Samela, H. Timko, K. Nordlund, S. Calatroni, M. Taborelli, and W. Wuensch, Crater formation by single ions, cluster ions and ion "showers", Nucl. Instrum. Methods Phys. Res., Sect. B 272, 374 (2012). 